\title{
Estructura de propiedad y capacidad informativa de los resultados contables*
}

\author{
Ownership structure and informativeness \\ of the accounting earnings
}

\author{
Domingo Javier Santana Martín. Universidad de Las Palmas de Gran Canaria \\ Carolina Bona Sánchez. Universidad de Las Palmas de Gran Canaria \\ Jerónimo Pérez Alemán. Universidad de Las Palmas de Gran Canaria
}

RESUMEN El presente trabajo analiza la relación entre la estructura de propiedad de las empresas no financieras cotizadas españolas en el periodo 1996-2002 y la capacidad informativa de sus resultados contables. Los resultados alcanzados muestran una relación negativa entre la participación en los derechos de voto del principal accionista y la capacidad informativa de los resultados contables. Sin embargo, no se obtienen resultados estadísticamente significativos en cuanto a la incidencia de la divergencia entre derechos de voto cash flow sobre la capacidad informativa de los resultados contables.

PALABRAS CLAVE Concentración de derechos de voto; Divergencia; Capacidad informativa.

ABSTRACT The present work analyzes the relationship between the ownership structure of listed nonfinancial Spanish companies and the informativeness of their accounting earnings, measured by the earnings-return relationship. We document that the informativeness of earnings decreases with the level of an ultimate owner's voting control. Nevertheless, we did not find statistically significant results concerning the relationship between the informativeness of accounting earnings and the extent to which the owner's voting rights exceed his cash flow rights. Our results are generally robust to controls for firm size, growth opportunities, leverage, and to the inclusion of only those companies with an ultimate owner.

KEYWORDS Ownership concentration; Divergence; Informativeness.

\section{INTRODUCCIÓN}

El sistema de gobierno corporativo constituye un mecanismo capaz de condicionar la calidad de la información contable. En este sentido, dado que dicha calidad no es un concepto unívoco, buena parte de los estudios que han abordado esta relación se han centrado en la variable resultado y sus diferentes atributos, tales como su tendencia a la manipulación o su nivel de conservadurismo [e.g., Leuz, Nanda y Wisocky, 2003; Xie, Davidson, DaDalt, 2003; García y Gill, 2004; Beekes, Pope y Young, 2004; García y Mora, 2004; Peasnell, Pope y Young, 2005; Cheng and Warfield, 2005; García, García y Mora, 2005; García, García y Peñalva, 2005].

$\left({ }^{\star}\right)$ Los autores agradecen las sugerencias realizadas a este trabajo por los participantes en el XII Foro de Finanzas celebrado en Barcelona los días 9 y 10 de diciembre de 2004 y organizado por el Centro de Investigación en Economía Financiera y Contabilidad y el Departamento de Economía y Empresa de la Universidad Pompeu Fabra, así como en el IV Workshop de Investigación Empírica en Contabilidad Financiera celebrado en Sevilla los días 3 y 4 de marzo de 2005 y organizado por el Departamento de Contabilidad y Economía Financiera de la Universidad de Sevilla y finalmente en el 28 Congreso anual organizado por la European Accounting Association celebrado en Göteborg (Suecia) los días 18 al 20 Mayo de 2005. Finalmente, los autores quieren agradecer especialmente las sugerencias realizadas en el proceso de revisión. 
En este contexto, un conjunto de trabajos ha centrado su atención en el análisis de la incidencia de la estructura de propiedad como mecanismo de gobierno en la calidad de la información contable. Así, en relación con la propiedad interna, Warfield, Wild y Wild [1995] ponen de manifiesto, para una muestra de empresas americanas, la existencia de una asociación positiva entre propiedad directiva y calidad de la información contable, medida tanto en términos de tendencia a la manipulación como de capacidad informativa. Sin embargo, en este mismo entorno, LaFond y Roychowdhory [2006] muestran una relación negativa entre propiedad interna y calidad de los resultados contables en términos de oportunidad y conservadurismo. Por su parte, Yeo, Tan, Ho y Chen [2002] obtienen evidencia de una relación no lineal entre propiedad interna y credibilidad de la información contable para una muestra de empresas cotizadas en Singapur. Más concretamente, tales autores concluyen que la capacidad informativa de los resultados contables se incrementa con la propiedad interna para niveles bajos de propiedad, mientras que para niveles altos, la propiedad interna resulta ineficiente como mecanismo de alineación de intereses.

De otra parte, Fan y Wong [2002], analizan la incidencia de la concentración de derechos de voto en la capacidad informativa de los resultados contables en el este asiático, incorporando en su estudio fundamentalmente países de origen legal anglosajón y germano, en los que el sistema legal proporciona a los accionistas minoritarios una alta e intermedia protección, respectivamente [La Porta, Lopez de Silanes, Shleifer y Vishny, 1998]. Sus resultados reflejan una relación negativa entre concentración de los derechos de voto en manos del principal accionista y la capacidad informativa de los resultados contables. En esta línea, en el contexto de las empresas cotizadas españolas, Azofra, Castrillo y Delgado [2003] constatan una relación positiva entre la manipulación contable y la concentración de propiedad por parte del accionista mayoritario.

Asimismo, la incidencia de la divergencia entre derechos de voto y cash flow en la calidad de la información contable ha sido analizada por Fan y Wong [2002], quienes encuentran que dicha separación incide negativamente en la capacidad informativa de los resultados contables. Este resultado se encuentra en consonancia con los obtenidos por Haw, Hu, Hwang y Wu [2004] quienes ponen de manifiesto, en un contexto internacional, una relación positiva entre la discrecionalidad directiva ejercida sobre los resultados y la divergencia entre derechos de voto y cash flow del principal accionista. En esta línea, Francis, Schipper y Vincent [2005] encuentran que la separación entre ambos derechos a través del uso de acciones con diferentes derechos de voto reduce la capacidad informativa de los resultados.

De esta forma, los trabajos precedentes han analizado la incidencia de la estructura de propiedad en la capacidad informativa de los resultados fundamentalmente en entornos institucionales que difieren de manera significativa del contexto en el que se desarrollan las actividades corporativas en España. Más concretamente, las relaciones contractuales de las empresas españolas se enmarcan en una estructura de gobierno propia de un país de origen legal francés, y por tanto, bajo un sistema legislativo que proporciona la menor defensa de los intereses de los inversores externos [La Porta et al., 1998] y que favorece, en mayor medida, una imagen de propiedad caracterizada por la elevada concentración de los derechos de voto en manos de los accionistas controladores y por la utilización de estructuras piramidales que permiten la separación entre derechos de voto y cash flow. 
De este modo, el presente trabajo tiene como objetivo analizar la incidencia de la estructura de propiedad de las empresas cotizadas españolas sobre la capacidad informativa de sus resultados contables. El análisis de esta influencia se ha realizado considerando el nivel de concentración de los derechos de voto en manos del principal accionista y el grado de divergencia entre sus derechos de voto y cash flow. Para ello, y en línea con trabajos precedentes, se utilizan dos argumentos que podrían justificar la incidencia de la estructura de propiedad en la capacidad informativa de los resultados contables. El primero, el efecto atrincheramiento [Morck, Shleifer y Vishny, 1988], derivado del conflicto entre propietarios mayoritarios y minoritarios, predice una influencia negativa de la citada concentración sobre la capacidad informativa de los resultados contables. No obstante, a medida que se incrementa la participación en la propiedad podría producirse un efecto alineamiento [Jensen y Meckling, 1976] que invierta el sentido de la relación anterior. El segundo, el efecto información [Jensen y Meckling, 1992; Christie, Joye y Watts, 2003] predice una incidencia negativa de la concentración de propiedad en la capacidad informativa de los resultados contables, ya que se considera la opacidad como una estrategia adecuada para evitar la transmisión de conocimiento relevante sobre las actividades productivas de la empresa a los competidores [Fan y Wong, 2002; Dyck y Zingales, 2004].

Asimismo, la presencia de estructuras contractuales que permiten la separación entre derechos de voto y cash flow podría intensificar el efecto atrincheramiento anteriormente referido, ya que en presencia de tales estructuras, el accionista controlador posee mayores niveles de voto con una menor participación en los derechos de propiedad, y por tanto, mayores incentivos para obtener beneficios privados [Fan y Wong, 2002; Haw et al., 2004; Francis et al., 2005].

Este trabajo extiende la literatura precedente en los siguientes aspectos. En primer lugar, analiza de modo directo la incidencia tanto de la concentración de derechos de voto en manos del principal accionista como de la divergencia entre sus derechos de voto y cash flow en la capacidad informativa de los resultados contables de las empresas cotizadas españolas. De otra parte, el estudio se acomete en un entorno institucional en el que el sistema legislativo proporciona la menor protección a los inversores externos. Asimismo, se utiliza una medida más completa para el análisis de la concentración de derechos de voto en manos del principal accionista mediante la determinación completa de las estructurales piramidales que permiten separar los derechos de voto y cash flow. Finalmente, para el análisis de la estructura de propiedad no se asume la hipótesis de que los derechos de voto en manos del último propietario no experimentan variaciones importantes a lo largo del tiempo, ya que se dibuja la estructura de propiedad para cada uno de los años objeto de estudio.

El resto del trabajo se estructura de la siguiente forma. En el segundo apartado abordamos los planteamientos teóricos que justifican la incidencia de la estructura de propiedad en la capacidad informativa de los resultados contables. Posteriormente, en el tercer apartado describimos los aspectos metodológicos que configuran el estudio empírico. Los resultados alcanzados se muestran en el cuarto apartado. Finalmente, exponemos las principales conclusiones del trabajo. 


\section{DESARROLLO DE LAS HIPÓTESIS}

\section{Concentración de propiedad y capacidad informativa}

Una gran parte de los trabajos enmarcados en el análisis de la estructura de propiedad se encuentran unidos a la imagen de propiedad difusa planteada por Berle y Means [1932]. Sin embargo, diversos estudios reflejan que en las grandes corporaciones de los países más desarrollados la presencia de estructuras de propiedad difusa no es tan general como predicen esos autores. Así, numerosos trabajos revelan la importancia de estructuras de propiedad concentrada en las que uno o muy pocos accionistas significativos ejercen un nivel de control destacado en las empresas cotizadas [e.g., Demsetz, 1983; Demsetz y Lehn, 1985; Shleifer y Vishny, 1986; Holderness y Sheehan, 1988; Galve y Salas, 1992; González y Menéndez, 1993; Andrés, Azofra y Rodríguez, 2000; López y Rodríguez, 2001;Holderness, Kroszner y Sheehan, 1999; La Porta, López-de-Silanes y Shleifer, 1999; Pedersen y Thomsen, 1999; Claessens, Djankov y Lang, 2000, y Faccio y Lang, 2002].

En este contexto, junto a los factores que explican la presencia de estructuras de propiedad concentrada, determinados, entre otros, por Fama y Jensen [1983], Demsetz [1983], Shleifer y Vishny [1986] y Denis y Denis [1994], un creciente cuerpo de estudios han centrando su atención en el entorno legal como factor determinante del sistema de gobierno, analizando de manera explícita el grado de eficacia del sistema legislativo en el control de las relaciones de agencia establecidas en las organizaciones, y especialmente, en la defensa de los intereses de los inversores externos. De esta manera, el nivel defensivo proporcionado por el entorno legal se configura como un elemento determinante de la estructura de propiedad utilizada por las empresas.

En esta línea, el estudio de Bebchuk [1999] sugiere una mayor presencia de estructuras de propiedad concentrada en los países donde la riqueza de los accionistas minoritarios se encuentra escasamente protegida por el sistema legal. Por su parte, Shleifer y Wolfenzon [2002] centran su estudio en la estructura del mercado de capitales, estableciendo que ante una mayor defensa de los intereses de los accionistas, dichos mercados estarán más desarrollados y existirá una menor concentración de propiedad. Estos argumentos teóricos se encuentran en la línea de los resultados empíricos alcanzados en los trabajos de La Porta et al. [1999], Claessens, Djankov y Lang [2000] y Faccio y Lang [2002].

En este sentido, aunque la presencia de estructuras de propiedad concentrada no limita la importancia de los costes de agencia, ya que sigue existiendo una divergencia de intereses entre propiedad y control, no es menos cierto que este nuevo contexto desplaza el conflicto derivado de las relaciones contractuales que se definen en el marco corporativo. De esta forma, los conflictos de agencia se trasladan de la divergencia entre accionistas minoritarios y directivos, propio de las estructuras de propiedad difusa [Jensen y Meckling, 1976, Fama, 1980 y Fama y Jensen, 1983], a la divergencia de intereses entre accionistas controladores y propietarios minoritarios [La Porta, López-de-Silanes, Shleifer, y Vishny, 2000].

En un contexto de propiedad difusa la relación entre propiedad interna y la calidad de la información contable ha sido abordada por diferentes autores. Warfield et al. [1995] muestran como la propiedad interna está positivamente relacionada con el poder explicativo de los resultados contables, ya que cuando los gestores poseen menor participación en el ca- 
pital de la empresa tienen mayores incentivos para incurrir en actuaciones no maximizadoras del valor. Por su parte, Yeo et al. [2002] señalan que la capacidad informativa de los resultados no siempre se incrementa con el aumento de la propiedad interna, ya que si bien para niveles bajos de propiedad es cierto que esta última se relaciona de modo positivo con la capacidad informativa de los resultados contables, para niveles altos de propiedad interna la relación entre ambas magnitudes se vuelve negativa.

De otra parte, en un entorno de propiedad concentrada, Fan y Wong [2002] analizan la incidencia de la propiedad sobre la capacidad informativa de los resultados contables a partir de dos argumentos que podrían explicar la relación entre ambas. En primer lugar, el atrincheramiento formulado por Morck et al. [1988], basado en la influencia del accionista controlador sobre la información suministrada por la empresa al mercado. De este modo, los agentes internos tienen incentivos para ocultar sus beneficios privados, ya que si dichos beneficios son detectados, los agentes externos podrían tomar acciones disciplinarias contra ellos [Zingales, 1994 y Shleifer y Vishny, 1997]. Sobre la base del argumento anterior, el inversor externo prestará escasa atención a la información contable, ya que espera que los objetivos de la misma sigan, en mayor medida, los intereses particulares del propietario mayoritario, más que el auténtico reflejo de las consecuencias económicas de las transacciones efectuadas por la empresa. No obstante, a medida que se incrementa el nivel de concentración de propiedad podría surgir un efecto alineamiento [Jensen y Meckling, 1976] que incida de forma positiva en la capacidad informativa de los resultados contables, ya que al aumentar la participación del accionista controlador se reducen los incentivos de dicho propietario a expropiar de la riqueza de los propietarios minoritarios.

Por otro lado, Fan y Wong [2002] y Dyck y Zingales [2004], a partir de los argumentos teóricos aportados por Jensen y Meckling [1992] y Christie et al. [2003], consideran el efecto información, basado en la limitación de la fuga de conocimiento específico sobre la gestión de la empresa a los competidores. Considerando este argumento, un entorno de propiedad concentrada podría fomentar una mayor opacidad y menor transparencia informativa, derivada del interés de accionistas mayoritarios y minoritarios por prestar la menor información posible al mercado. Por tanto, el efecto información asociado a la concentración de propiedad afectará negativamente a la capacidad informativa de los resultados contables.

Los argumentos expuestos con relación a la incidencia de la concentración de propiedad sobre la capacidad informativa de los resultados contables suponen que mientras los efectos atrincheramiento e información pueden afectar negativamente a la capacidad informativa de los resultados contables, el efecto alineamiento puede incrementarla. Por tanto, el presente trabajo tiene como primer objetivo contrastar empíricamente cuál de los efectos anteriores domina en el contexto de las empresas cotizadas españolas, para lo cual se formulan las siguientes hipótesis alternativas:

$H_{1}$ : La concentración de derechos de voto en manos del principal accionista incidirá en la capacidad informativa de los resultados contables.

$H_{1 a}$ : La concentración de derechos de voto en manos del principal accionista incidirá negativamente (efecto atrincheramiento/información) en la capacidad informativa de los resultados contables.

$H_{16}$ : La concentración de derechos de voto en manos del principal accionista incidirá positivamente (efecto alineamiento) en la capacidad informativa de los resultados contables. 


\section{Divergencia entre derechos de voto y cash flow y capacidad informativa}

La Porta et al. [1999] muestran que las grandes empresas de los países más desarrollados se encuentran controladas por accionistas que poseen mayores derechos de voto que de cash flow. Dicha separación es posible mediante la emisión de acciones con diferentes niveles de voto, el uso de pirámides o la presencia de estructuras de propiedad cruzadas. En este sentido, los autores citados señalan que este tipo de estructuras es más frecuente en países en los que el sistema legal proporciona una menor defensa de los intereses de los inversores externos, configurándose las pirámides como el principal mecanismo utilizado por los accionistas controladores para separar derechos de voto y cash flow.

Bebchuk, Kraakman y Triantis [2000] argumentan que la utilización de estructuras de propiedad que permiten la separación de derechos de voto y cash flow hace posible que se encuentren de manera combinada los problemas de agencia asociados a las estructuras de propiedad concentrada y difusa. De esta forma, las estructuras que permiten separar propiedad y control se asimilan a las de propiedad concentrada por el hecho de que aislan a los accionistas controladores de la disciplina del mercado de control corporativo, y se asemejan a su vez a las de propiedad dispersa porque delegan el control corporativo en un accionista que posee una pequeña fracción de los derechos de cash flow.

En este sentido, Fan y Wong [2002] y Francis et al. [2005] señalan que la separación entre derechos de voto y cash flow puede agravar el efecto atrincheramiento, ya que los agentes internos, al poseer menores derechos de cash flow que de voto, tienen mayores incentivos para adquirir beneficios privados en detrimento de la riqueza de los accionistas minoritarios. En esta línea, Harris y Raviv [1988] y Grossman y Hart [1988] argumentan que la separación entre derechos de voto y cash flow podría conllevar una menor transparencia informativa, consistente con el efecto atrincheramiento. Este hecho, junto con la menor fuerza del resto de mecanismos de gobierno corporativo, acentúa los incentivos a la expropiación por parte de los propietarios controladores, todo lo cual podría traducirse en una disminución de la capacidad informativa de los resultados contables. De este modo, se formula la siguiente hipótesis:

$H_{2}$ : La divergencia entre derechos de voto y cash flow en manos del principal accionista incidirá negativamente en la capacidad informativa de los resultados contables.

\section{ASPECTOS METODOLÓGICOS}

\section{Muestra}

La determinación de la muestra parte de las 117 empresas no financieras que cotizaban en el mercado español a finales de 2002, de las cuales se eliminaron, por un lado, una sociedad que se encontraba suspendida de cotización al hallarse en proceso de liquidación, y por otro, seis empresas no domiciliadas en España. Por último, no se consideraron nueve empresas por no contar con al menos tres observaciones en el periodo 1996-2002, y 2 sociedades excluidas de cotización durante el año 2003, por lo que la muestra final quedó constituida por 99 empresas. 


\section{Definición de las cadenas de control}

La metodología utilizada para analizar la estructura de propiedad parte de la clasificación de las empresas en dos grupos principales, por un lado las empresas de propiedad difusa, y por otro las que poseen un último propietario. En el presente trabajo, siguiendo a La Porta et al. [1999], Claessens et al. [2000] y Faccio y Lang [2002], diremos que una empresa tiene un último propietario cuando el principal accionista de la misma posea directa $o$ indirectamente un porcentaje de participación en los derechos de voto igual o superior a un nivel de control establecido, que en nuestro caso será igual al $10 \%{ }^{(1)}$.

La utilización de la especificación única de los eslabones inmediatos de propiedad presenta dos inconvenientes. De una parte, puede otorgar un nivel de participación a un accionista que no se corresponde con el que realmente ostenta, y de otra, puede situar en el control de una determinada compañía a un agente que realmente no se encuentra en la posición final de las relaciones de propiedad. Tales inconvenientes se solucionan mediante la metodología de definición de las cadenas de control aplicada en este estudio, basada en la determinación de los eslabones de propiedad de una empresa, de forma que cuando las acciones de una compañía están en manos de otra sociedad, se examina la propiedad de los derechos de voto de esta última, identificando a su principal accionista y continuando con este mismo proceso hasta llegar al último propietario de los derechos de voto. Esta forma de proceder permite dibujar de manera más precisa las relaciones contractuales entre los diferentes propietarios de cada empresa, ya que define en su totalidad el nivel de control que ejerce el principal accionista a través del entramado de sociedades que pudieran existir entre éste y la empresa objeto de estudio. Además de ello, dibujar la cadena de control de una empresa posibilita el análisis de estructuras de propiedad que permiten al principal accionista obtener divergencias entre sus derechos de voto y cash flow, variable fundamental en entornos de propiedad concentrada para el análisis de los conflictos de agencia que pueden existir entre dicho accionista y el resto de propietarios ${ }^{(2)}$.

Con el objetivo de determinar las relaciones de propiedad-control sobre la base de la metodología de las cadenas de control expuesta, en una primera fase partimos de la información sobre participaciones significativas ofrecida por la Comisión Nacional del Mercado de Valores (CNMV), de la que se obtuvieron las participaciones directas e indirectas de aquellos accionistas con un porcentaje de acciones superior al 5\%, así como de la propiedad en

(1) La Porta et al. [1999], Claessens et al. [2000] y Faccio y Lang [2002] también utilizan el 20\% como nivel de control. No obstante, en este trabajo presentamos únicamente los resultados para un nivel de control del $10 \%$, ya que los resultados empíricos no experimentan cambios significativos entre ambos niveles de control. Además, la utilización de un nivel de control del $10 \%$ se adecua en mayor medida a la realidad de las relaciones de propiedad de las empresas cotizadas españolas.

(2) De este modo, si en la empresa A el principal accionista (I) posee un $16 \%$ de sus derechos de voto, y la empresa $A$ es propietaria al mismo tiempo del $20 \%$ de la empresa B, diremos que la empresa B está controlada por I, para un nivel de control del $10 \%$, ya que tiene un último propietario, quien controla B indirectamente a través de la empresa A. En el ejemplo anterior, el accionista I posee un 3,2\% de los derechos de cash flow de B, es decir, el producto de sus participaciones a lo largo de la cadena $(0,16 \times 0,2)$. Sin embargo posee, siguiendo la metodología propuesta por Claessens et alt. [2000] y Faccio y Lang [2002], el 16 por ciento de los derechos de voto de B, es decir, el enlace más débil de la cadena de control [mín $(0,16 ; 0,2)]$. Por tanto, si el accionista I posee el 100 por cien de los derechos de voto de A, no existe una divergencia entre derechos de control y cash flow, ambos derechos supondrían en este caso un $20 \%$. En ocasiones observamos que una empresa se encuentra controlada a través de una cadena de control múltiple, es decir, cuando el último propietario controla la empresa a través de diversas cadenas de control. Así, si en el ejemplo anterior el accionista I posee directamente un $6 \%$ de los derechos de voto de B, entonces dicho propietario participa en el 9,2\% de los derechos de cash flow de $B(0,16 \times 0,2+0,06)$, y en el $22 \%$ de sus derechos de voto, es decir, $[(\operatorname{mín}(0,16 ; 0,2)+0,06)]$ 
manos de los consejeros, independientemente del montante de la misma. En una segunda fase, se complementó dicha fuente con la base de datos Informa, que ofrece información sobre la propiedad y el consejo de administración de sociedades españolas cotizadas y no cotizadas, datos que fueron necesarios para completar en su totalidad la cadena de control de una empresa, es decir, para identificar su último propietario. En aquellos casos en los que encontramos sociedades no residentes en España como accionistas de empresas españolas, completamos sus estructuras de propiedad a través de las memorias obtenidas en las páginas web de dichas sociedades, consultando mediante correo electrónico las dudas que surgieron para dibujar su cadena de control.

Por tanto, este estudio presenta dos diferencias con relación a los trabajos precedentes que utilizan la metodología de definición de las cadenas de control. En primer término, se dibuja la totalidad de los eslabones de propiedad de las empresas analizadas, ya que se identifica a los propietarios de las sociedades no cotizadas que se encuentran en los eslabones intermedios. En segundo lugar, no se asume la hipótesis de que las estructuras de propiedad no cambian a lo largo del tiempo, al determinarse las cadenas de control en cada uno de los años objeto de estudio ${ }^{(3)}$.

En la Tabla 1 se muestran los resultados obtenidos en relación con los derechos de voto y cash flow en manos del principal accionista. Así, se puede observar en el panel A como los derechos de cash flow, en términos de mediana, se encuentran en torno al $25 \%$, frente al 29 por ciento de los derechos de voto que se recogen en el panel B. Este hecho determina, por un lado, la elevada concentración de propiedad y control en manos del principal accionista, y por otro, que este propietario posee un mayor nivel de derechos de voto en comparación con sus derechos de cash flow. Por otro lado, en el panel C se observa que la relación propiedad-control del principal accionista sigue una evolución decreciente, lo que implica que en España la divergencia entre derechos de voto y de cash flow ha aumentado entre los años 1996 y 2002, aunque la mediana de dicha relación es 1 en todo el periodo ${ }^{(4)}$.

TABLA 1

DERECHOS DE VOTO Y CASH FLOW DEL PRINCIPAL ACCIONISTA DE LAS EMPRESAS COTIZADAS ESPAÑOLAS

\begin{tabular}{|cccccc|}
\hline \multicolumn{7}{c|}{ Panel $A}$. & DERECHOS DE cash flow \\
\hline \multicolumn{7}{c}{ Media } & Mediana & Desviación & $1 .{ }^{\circ}$ Cuartil & $3 .{ }^{\circ}$ Cuartil \\
\hline 1996 & 33,5 & 25,1 & 22,5 & 14,1 & 50,5 \\
1997 & 31,5 & 25,0 & 21,7 & 14,5 & 48,2 \\
1998 & 30,9 & 26,5 & 21,6 & 12,5 & 49,1 \\
1999 & 31,3 & 26,7 & 22,4 & 11,2 & 50,4 \\
2000 & 32,3 & 26,9 & 23,0 & 11,4 & 50,4 \\
2001 & 31,6 & 27,7 & 22,9 & 10,9 & 49,4 \\
2002 & 31,9 & 24,9 & 24,7 & 10,5 & 49,4 \\
\hline
\end{tabular}

(Continúa pág. sig.)

(3) Para llevar a cabo su estudio, Fan y Wong [2002] culminan las cadenas de control cuando se ha identificado al accionista que detenta el $50 \%$ de los derechos de voto, realizando además la presunción de que las estructuras de propiedad no varían a lo largo del tiempo.

(4) La estructura de propiedad utilizada por los propietarios finales de las empresas analizadas, con el fin de lograr separar derechos de voto y cash flow, se basa exclusivamente en la utilización de estructuras piramidales, ya que el empleo de acciones con distintos derechos de voto y el uso de cross-holding es nulo. 
TABLA 1 (Cont.)

DERECHOS DE VOTO Y CASH FLOW DEL PRINCIPAL ACCIONISTA DE LAS EMPRESAS COTIZADAS ESPAÑOLAS

\begin{tabular}{|c|c|c|c|c|c|}
\hline \multicolumn{6}{|c|}{ Panel B. Derechos de Voto } \\
\hline & Media & Mediana & Desviación & $1 .^{\circ}$ Cuartil & 3. ${ }^{\circ}$ Cuartil \\
\hline 1996 & 35,4 & 28,1 & 22,4 & 15,0 & 51,2 \\
\hline 1997 & 33,6 & 29,5 & 21,4 & 15,8 & 50,2 \\
\hline 1998 & 33,6 & 29,5 & 21,5 & 15,2 & 50,2 \\
\hline 1999 & 33,8 & 28,3 & 22,5 & 14,7 & 53,5 \\
\hline 2000 & 35,2 & 28,8 & 23,5 & 14,2 & 54,0 \\
\hline 2001 & 34,9 & 29,7 & 23,5 & 13,7 & 51,0 \\
\hline 2002 & 35,0 & 29,1 & 24,4 & 14,9 & 51,2 \\
\hline \multicolumn{6}{|c|}{ Panel C. CASH FLow/Votos } \\
\hline & Media & Mediana & Desviación & $1 .^{\circ}$ Cuartil & 3. ${ }^{\circ}$ Cuartil \\
\hline 1996 & 0,94 & 1 & 0,15 & 1 & 1 \\
\hline 1997 & 0,94 & 1 & 0,17 & 1 & 1 \\
\hline 1998 & 0,92 & 1 & 0,20 & 1 & 1 \\
\hline 1999 & 0,93 & 1 & 0,18 & 1 & 1 \\
\hline 2000 & 0,91 & 1 & 0,19 & 1 & 1 \\
\hline 2001 & 0,91 & 1 & 0,20 & 0,99 & 1 \\
\hline 2002 & 0,89 & 1 & 0,22 & 0,98 & 1 \\
\hline
\end{tabular}

La muestra está compuesta por 99 empresas no financieras cotizadas españolas entre 1996 y 2002.

Para la determinación del principal accionista se ha seguido la metodología de definición del propietario final de La Porta et al. [1999], Claessens et al. [2000] y Faccio y Lang [2002]. De esta forma, una empresa tiene un último propietario cuando el principal accionista de la misma posee directa 0 indirectamente un porcentaje de participación en los derechos de voto igual o superior a un nivel de control establecido, que en este trabajo es igual al $10 \%$.

La ratio derechos de cash flow sobre derechos de voto posee un rango de valores entre 0 y 1.

\section{Definición de variables}

Variable endógena.-La rentabilidad de las acciones $C A R_{i}$, nos permite analizar la capacidad informativa de los resultados contables, siendo medida a través de la rentabilidad acumulada ajustada por la evolución del mercado de la empresa $i$ en el año $t$. La rentabilidad de las acciones es calculada, siguiendo a Fan y Wong [2002], de manera continua con los precios doce meses antes del último día para la presentación de las cuentas anuales según la normativa bursátil ${ }^{(5)}$. Los datos utilizados para la construcción de dicha variable han sido las series históricas de cotización proporcionadas por la Sociedad de Bolsas.

Variables explicativas.-El análisis de la capacidad informativa de los resultados contables se realiza tradicionalmente a partir de la magnitud del coeficiente estimado en la regresión entre la rentabilidad de las acciones y los resultados contables - earnings response coefficient - [Teoh y Wong, 1993; Imhoff y Lobo, 1992; Warfield et al., 1995; Subramanyan y Wild, 1996; Fan y Wong, 2002; Gul y Wah, 2002; Yeo et al., 2002]. En este estudio, para el análisis de la capacidad informativa de los resultados contables, se utiliza en línea con la

(5) En aquellas empresas en las que el año fiscal no coincide con el final de año natural se calcularon las rentabilidades teniendo en cuenta la fecha de cierre del ejercicio contable. 
literatura precedente $\mathrm{NI}_{\mathrm{i}}$, es decir, el resultado neto de la empresa $i$ en el año $t$ dividido por el valor de mercado de las acciones al principio del año $t$.

Por su parte, las variables utilizadas para el análisis del efecto de la estructura de propiedad en la capacidad informativa de los resultados contables son, en primer lugar, la incidencia de los derechos de voto $V_{i}$, medida a través del porcentaje de derechos de voto en manos del mayor propietario de la empresa $i$ en el año $t$. En segundo lugar, se utiliza el efecto de la separación entre derechos de voto y cash flow $C V_{i}$, medida a través de la ratio derechos de cash flow sobre derechos de voto del principal accionista de la empresa $i$ en el año $t$. Tal como puede apreciarse, $C V_{i}$ está inversamente relacionada con la divergencia entre derechos de voto y cash flow.

Variables de control.-Con el objetivo de analizar la posible incidencia de otras características corporativas en la capacidad informativa de los resultados contables, se ha introducido, en línea con la literatura previa, una serie de variables de control. Así, con el fin de considerar el efecto de las oportunidades de crecimiento en el análisis de la relación resultados-rentabilidad se incluye en el modelo $Q_{i}$, que mide la relación existente entre el valor de mercado de las acciones y el valor contable de los activos al principio del año $t$. Por otro lado, se incorpora en el análisis el nivel de endeudamiento, $L E V_{i}$, medido a través de la relación entre el valor contable de la deuda y el total de activos al principio del año $t$, con el objetivo de recoger el riesgo derivado de la utilización de recursos ajenos, de forma que se espera una relación inversa entre el nivel de endeudamiento y la capacidad informativa de los resultados contables. Por último, se ha incluido la dimensión de la empresa, $S I Z E_{i}$, medida a través del logaritmo del valor de mercado de las acciones, en miles de euros, al principio del año t, al objeto de considerar otros factores no considerados que pudiesen afectar a la relación resultados-rentabilidad.

Los estadísticos descriptivos, así como la matriz de correlaciones de las variables consideradas, se muestran en la Tabla 2 . En ella se observa que la variable $C A R$ tiene un valor medio de 0,120 mientras que la medida representativa de los resultados contables $N I$, posee un valor medio de 0,016 . Los valores estadísticos que presentan las variables de propiedad $V$ y $C V$ son consistentes con los presentados en la tabla 1. Así, el propietario final posee, en términos medios, un $34,52 \%$ de los derechos de voto, detentando en términos promedios mayores derechos de voto que de cash flow. De esta forma, los accionistas controladores poseen en media un $8,2 \%$ menos de derechos de cash flow que de voto, al ser el valor medio de la ratio 0,918. La matriz de correlaciones no muestra valores elevados entre las variables explicativas, hecho que nos permite suponer que sea improbable la presencia de problemas de multicolinealidad en la especificación de los modelos de regresión.

TABLA 2

ESTADíSTICOS DESCRIPTIVOS Y MATRIZ DE CORRELACIÓN

\begin{tabular}{|lccccccc|}
\hline & CAR & NI & $V$ & $C V$ & SIZE & LEV & $Q$ \\
\hline Media & 0,120 & 0,016 & 34,52 & 0,918 & 13,28 & 0,644 & 1,295 \\
Mediana & 0,014 & 0 & 29,51 & 1 & 13 & 0,565 & 0,719 \\
Desviación & 0,402 & 0,211 & 22,79 & 0,190 & 1,696 & 0,562 & 2,916 \\
Mínimo & $-2,057$ & $-3,994$ & 0 & 0,052 & 10 & 0,034 & 0,050 \\
Máximo & 1,216 & 1 & 99,5 & 1 & 18,03 & 8,592 & 57,82 \\
\hline
\end{tabular}


TABLA 2 (Cont.)

ESTADÍSTICOS DESCRIPTIVOS Y MATRIZ DE CORRELACIÓN

\begin{tabular}{|c|c|c|c|c|c|c|c|}
\hline \multicolumn{8}{|c|}{ Matriz de CORRELACIÓN } \\
\hline & CAR & $N I$ & V & $\mathrm{CV}$ & SIZE & LEV & $Q$ \\
\hline CAR & 1 & & & & & & \\
\hline $\mathrm{NI}$ & 0,211 & 1 & & & & & \\
\hline V & 0,015 & 0,014 & 1 & & & & \\
\hline CV & $-0,066$ & $-0,062$ & 0,022 & 1 & & & \\
\hline SIZE & 0,114 & 0,103 & $-0,007$ & $-0,125$ & 1 & & \\
\hline LEV & $-0,039$ & 0,013 & 0,023 & 0,070 & 0,039 & 1 & \\
\hline$Q$ & 0,034 & 0,005 & 0,004 & 0,028 & 0,158 & 0,516 & 1 \\
\hline
\end{tabular}

Definición de variables:

$C A R_{i}$, rentabilidad acumulada ajustada por la evolución del mercado de la empresa $i$ en el año $t$. La rentabilidad de las acciones es calculada de manera continua con los precios doce meses antes del último día para la presentación de las cuentas anuales según la normativa bursátil española. $N I_{i}$, el resultado neto disponible para los accionistas de la empresa $i$ en el año t dividido por el valor de mercado de las acciones al principio del año $t$. Vi, porcentaje de derechos de voto en manos del mayor propietario de la empresa i en el año $t$. $C V_{i}$, ratio derechos de cash flow sobre derechos de voto del principal accionista de la empresa $i$ en el año $t$. $Q_{i}$, relación entre el valor de mercado de las acciones y el valor contable de los activos al principio del año $t$. $L E V_{i}$, relación entre el valor contable de la deuda y el total de activos al principio del año $t$. $S I Z E_{i j}$ logaritmo del valor de mercado de las acciones, en miles de euros, al principio del año $t$.

La muestra está constituida por 99 empresas no financieras cotizadas españolas entre 1996 y 2002.

\section{ANÁLISIS DE RESULTADOS}

La relación básica entre la rentabilidad de las acciones en el mercado y los resultados contables se especifica en el siguiente modelo:

$$
C A R_{i}=\alpha_{0}+\beta_{1} N I_{i}+\mu_{i}
$$

Por otro lado, el estudio de la incidencia en la capacidad informativa de los resultados contables del nivel de concentración de derechos de voto, así como del grado de divergencia entre derechos de voto y cash flow en manos del principal accionista se realiza en base al modelo de regresión propuesto por Fan y Wong [2002], a partir de la siguiente especificación:

$$
C A R_{i}=\alpha_{0}+\beta_{1} N I_{i}+\beta_{2} N I_{i} V_{i}+\beta_{3} N I_{i} C V_{i}+\beta_{4} N I_{i} S I Z E_{i}+\beta_{5} N I_{i} Q_{i}+\beta_{6} N I L e v_{i}+\mu_{i}
$$

En la Tabla 3 se muestran los resultados de las regresiones realizadas con el objetivo de contrastar la incidencia de la estructura de propiedad en la capacidad informativa de los resultados usando un pool formado por 605 observaciones empresa-año. La significación estadística de los coeficientes está basada en la matriz de covarianzas de White [1980].

De forma previa al estudio de la incidencia de la estructura de propiedad en la capacidad informativa de los resultados contables, se analiza si en el mercado de capitales español los resultados contables juegan un papel relevante en la determinación de rentabilidad de los acciones. Tal como se aprecia en la Eq. (1) los resultados revelan una relación positiva y estadísticamente significativa entre ambas variables, reflejo de la incidencia de la información contable en la rentabilidad de las acciones. 
TABLA 3

ESTRUCTURA DE PROPIEDAD Y CAPACIDAD INFORMATIVA DE LOS RESULTADOS CONTABLES

\begin{tabular}{|lcc|}
\hline & $(1)$ & $(2)$ \\
\hline $\mathrm{C}$ & 0,005 & $-0,021$ \\
$\mathrm{NI}$ & $(0,35)$ & $(-1,01)$ \\
$\mathrm{NI} \times \mathrm{V}$ & $0,402^{* \star *}$ & $-0,242$ \\
$\mathrm{NI} \times \mathrm{CV}$ & $(7,47)$ & $(-0,16)$ \\
$\mathrm{NI} \times \mathrm{SIZE}$ & & $-0,006^{*}$ \\
& & $(-1,73)$ \\
$\mathrm{NI} \times \mathrm{Q}$ & & $-1,204$ \\
$\mathrm{NI} \times \mathrm{LEV}$ & & $(-1,61)$ \\
& & $0,176^{*}$ \\
\hline $\mathrm{R}-\mathrm{Sq}$ & & $(1,87)$ \\
$\mathrm{F}$ & & $0,866^{*}$ \\
$\mathrm{~N} .{ }^{0}$ observaciones & & $(1,66)$ \\
& & $-0,624^{\star \star *}$ \\
& 0,043 & $(-2,68)$ \\
\hline
\end{tabular}

Definición de variables:

$C A R_{p}$ rentabilidad acumulada ajustada por la evolución del mercado de la empresa $i$ en el año $t$. La rentabilidad de las acciones es calculada de manera continua con los precios doce meses antes del último día para la presentación de las cuentas anuales según la normativa bursátil española. $N l_{i}$, el resultado neto disponible para los accionistas de la empresa $i$ en el año $t$ dividido por el valor de mercado de las acciones al principio del año $t . V_{i}$, porcentaje de derechos de voto en manos del mayor propietario de la empresa $i$ en el año $t$. $C V_{p}$, ratio derechos de cash flow sobre derechos de voto del principal accionista de la empresa $i$ en el año $t$. $Q_{p}$ relación entre el valor de mercado de las acciones y el valor contable de los activos al principio del año $t$. $L E V_{i}$, relación entre el valor contable de la deuda y el total de activos al principio del año $t$. $S I Z E_{i}$ logaritmo del valor de mercado de las acciones, en miles de euros, al principio del año $t$.

La muestra está constituida por 99 empresas no financieras cotizadas españolas entre 1996 y 2002.

***, ${ }^{* *},{ }^{*}$ : Estadísticamente significativo al 1,5 y $10 \%$, respectivamente.

Estadístico $t$ ajustado de White entre paréntesis.

Por su parte, con relación a la incidencia de la estructura de propiedad en la credibilidad de los resultados contables, en la Eq. (2) se muestra como la concentración de derechos de voto en manos del principal accionista $(V$ ) tiene un efecto negativo y estadísticamente significativo en la capacidad informativa de los resultados contables, en consonancia con la hipótesis $H_{1} a$. De esta forma, los resultados sugieren que cuanto mayor es la proporción de derechos de voto en manos del principal accionista, menor será la credibilidad de los resultados contables en el mercado, lo cual es consistente con los efectos atrincheramiento y/o información asociados al nivel de concentración en el control de las empresas.

Por otro lado, la estimación del efecto de la divergencia entre derechos de voto y cash flow $(C V)$ revela una relación estadísticamente no significativa de la separación entre ambos derechos y la capacidad informativa de los resultados contables. Así pues, los resultados alcanzados no permiten aceptar la hipótesis $H_{2}$ que predice un efecto negativo en la capacidad informativa asociada a la utilización de estructuras piramidales, por lo que en el caso de las empresas cotizadas españolas, la credibilidad otorgada por el mercado a la información contable se reduce a medida que aumenta el nivel de control del principal accionista, no viéndose afectada por la utilización de estructuras que permiten separar los derechos de voto y cash flow. 
Con respecto a las variables de control, los resultados muestran un efecto negativo y estadísticamente significativo del nivel de endeudamiento en la capacidad informativa de los resultados contables. Esta relación inversa puede ser reflejo de la menor credibilidad otorgada por el mercado a la información contable a medida que aumenta el riesgo de insolvencia derivado del incremento de la financiación ajena [Dhaliwal, Kyung y Fargher, 1991]. Esto adquiere una especial importancia en el contexto de un país en el que la financiación bancaria se erige como principal mecanismo de captación de recursos. Por otro lado, tanto el tamaño de las empresas como la presencia de oportunidades de crecimiento afectan de manera positiva y estadísticamente significativa a la credibilidad de la información contable ofrecida por las empresas cotizadas españolas, en consonancia con los resultados obtenidos, para otros contextos geográficos, por Collin y Khotari [1989] Fan y Wong [2002] y Francis et al. [2005].

\section{Análisis de robustez}

Con el objeto de analizar la robustez de los resultados alcanzados, se ha realizado la regresión de los modelos especificados en el estudio de la estructura de propiedad, considerando únicamente las empresas en las que identificamos un propietario final de los derechos de voto, es decir, en aquellas en las que $V$ es mayor o igual al 10\%. Los resultados alcanzados se muestran en la Tabla 4, en la que se puede observar que las regresiones no cambian de manera cualitativa las conclusiones alcanzadas cuando se ha utilizado la totalidad de las empresas. Finalmente, se realizaron las regresiones utilizando como medida alternativa de $C A R$ la rentabilidad continua ajustada por el mercado de las acciones en una ventana de seis meses antes y después del final del año fiscal. Los resultados de las regresiones efectuadas sobre esta variable endógena muestran un efecto estadísticamente no significativo de la estructura de propiedad en la capacidad informativa de los resultados contables $^{(6)}$. Este resultado podría significar que el efecto de la información contable en la rentabilidad de las acciones se encuentra vinculada a la fecha en la que dicha información es revelada y no al periodo económico al que alude.

\section{TABLA 4}

ESTRUCTURA DE PROPIEDAD Y CAPACIDAD INFORMATIVA DE LOS RESULTADOS CONTABLES. EMPRESAS DE PROPIEDAD CONCENTRADA ${ }^{\text {a }}$

\begin{tabular}{|lcc|}
\hline & $(1)$ & $(2)$ \\
\hline $\mathrm{C}$ & 0,011 & $-0,018$ \\
$\mathrm{NI}$ & $(0,66)$ & $(-0,77)$ \\
& $0,389^{* * *}$ & $-1,277$ \\
$\mathrm{NI} \times \mathrm{V}$ & $(7,64)$ & $(-0,67)$ \\
& & $-0,006^{*}$ \\
$\mathrm{NI} \times \mathrm{CV}$ & & $(1,66)$ \\
$\mathrm{NI} \times \mathrm{SIZE}$ & & $-0,995$ \\
& & $(-1,32)$ \\
& & $0,240^{*}$ \\
\hline
\end{tabular}

(Continúa pág. sig.)

(6) Los resultados de las regresiones no ofrecidos en tablas, están disponibles para aquellos que los requieran a cualquiera de los autores. 
TABLA 4 (Cont.)

ESTRUCTURA DE PROPIEDAD Y CAPACIDAD INFORMATIVA DE LOS RESULTADOS CONTABLES. EMPRESAS DE PROPIEDAD CONCENTRADA ${ }^{\text {a }}$

\begin{tabular}{|lcc|}
\hline & $(1)$ & $(2)$ \\
\hline $\mathrm{NI} \times \mathrm{Q}$ & & 0,886 \\
$\mathrm{NI} \times \mathrm{LEV}$ & & $(1,57)$ \\
& & $-0,634^{* *}$ \\
\hline $\mathrm{R}-\mathrm{Sq}$ & & $(-2,41)$ \\
$\mathrm{F}$ & 0043 & 0,084 \\
$\mathrm{~N} .{ }^{\circ}$ observaciones & $58,31^{* * *}$ & $29,76^{* \star *}$ \\
\hline
\end{tabular}

Definición de variables:

CARi, rentabilidad acumulada ajustada por la evolución del mercado de la empresa i en el año $t$. La rentabilidad de las acciones es calculada de manera continua con los precios doce meses antes del último día para la presentación de las cuentas anuales según la normativa bursátil española. $N I_{i}$, el resultado neto disponible para los accionistas de la empresa $i$ en el año $t$ dividido por el valor de mercado de las acciones al principio del año $t$. $V_{i}$, porcentaje de derechos de voto en manos del mayor propietario de la empresa $i$ en el año $t$. $C V_{p}$, ratio derechos de cash flow sobre derechos de voto del principal accionista de la empresa $i$ en el año $t$. $Q_{p}$ relación entre el valor de mercado de las acciones y el valor contable de los activos al principio del año $t$. $L E V_{i}$, relación entre el valor contable de la deuda y el total de activos al principio del año $t$. $S I Z E_{i}$ logaritmo del valor de mercado de las acciones, en miles de euros, al principio del año $t$.

La muestra está constituida por 99 empresas no financieras cotizadas españolas entre 1996 y 2002.

a $L a$ muestra final excluye aquellas observaciones en las que $V<10 \%$.

***,****: Estadísticamente significativo al 1,5 y $10 \%$, respectivamente.

Estadístico $t$ ajustado de White entre paréntesis.

\section{CONCLUSIONES}

La creciente preocupación por la incidencia del sistema de gobierno corporativo, y en particular de la estructura de propiedad, sobre la capacidad informativa de los resultados contables, así como la escasez de trabajos que analicen la citada interrelación en España, ofrece la oportunidad de acometer este estudio en el contexto de un país que difiere de los entornos institucionales en los que se han centrado los trabajos precedentes que han estudiado dicha relación.

De este modo, el presente estudio se aborda en un contexto institucional de origen legal francés, en el que los intereses de los inversores externos se encuentran escasamente defendidos por el sistema legal, y en el que la imagen de propiedad viene determinada, entre otros aspectos, por la presencia de una elevada concentración de los derechos de voto en manos del principal accionista y la utilización de estructuras piramidales que permiten la divergencia entre derechos de voto y cash flow.

Los resultados alcanzados muestran una incidencia negativa de la concentración de derechos de voto en manos del principal accionista sobre la capacidad informativa de los resultados contables. Esto, podría evidenciar que a medida que aumenta el control ejercido por el accionista principal, sus intereses estarán menos alineados con los propietarios minoritarios, por lo que de acuerdo con el efecto atrincheramiento aumentarían sus incentivos para la obtención de beneficios privados. Consecuentemente, el mercado podría entender que, dado que el accionista principal controla el proceso de elaboración y divulgación de información contable, ésta podría alejarse del fiel reflejo de las operaciones desempeñadas, al objeto de ocultar la posible expropiación realizada. Asimismo, los resul- 
tados obtenidos también podrían venir explicados por la existencia de un efecto información, intensificado ante la presencia de estructuras de propiedad concentrada. En este sentido, la incidencia negativa de la participación en los derechos de voto del principal accionista sobre la capacidad informativa de los resultados contables puede ser reflejo de una tendencia a la opacidad en el proceso de revelación de información contable corporativa. Así, en el presente trabajo los resultados sobre la incidencia de la estructura de propiedad en la calidad de la información contable se encuentran en consonancia con los alcanzados por Fan y Wong [2002] y Azofra et al., [2003], y difieren de los obtenidos en un contexto de propiedad atomizada por Warfield et al. [1995] y Yeo et al. [2002].

Por otro lado, los resultados muestran una incidencia estadísticamente no significativa sobre la capacidad informativa de los resultados contables de la divergencia entre derechos de voto y cash flow en manos del principal accionista. De esta forma, la utilización por parte de los accionistas controladores de estructuras piramidales no parece afectar a la capacidad informativa en el caso de las empresas cotizadas españolas. Este resultado difiere del alcanzado por Fan y Wong [2002], quienes contrastan un efecto negativo de la divergencia entre derechos de propiedad y control en la credibilidad de la información contable. Una posible justificación a tal divergencia puede encontrarse en la diferencia de estructuras utilizadas en los referidos contextos para separar ambos derechos. Así, mientras en el este asiático es frecuente el uso del cross-holding [Claessens et al., 2000], en el caso español dicha estructura contractual no es utilizada, siendo las pirámides la estructura a la que recurren las empresas españolas para separar derechos de propiedad y control. Este bajo uso del cross-holding es una característica común entre las sociedades cotizadas en Europa, ya que como muestran Faccio y Lang [2002], tan sólo el 0,51\% de las empresas europeas lo utilizan. En este sentido, Bebchuck et al. [2000] argumentan que la estructura cross-holding refuerza el atrincheramiento de los accionistas significativos, ya que la diferencia principal con la estructura piramidal es que en el cross-holding los derechos de voto utilizados para controlar al grupo se distribuyen a través del mismo y no se concentran en manos de una única compañía o accionista.

De esta forma, el presente trabajo evidencia que la credibilidad otorgada por el mercado de capitales a la información contable viene determinada de modo significativo por la estructura de propiedad, y más concretamente, por el nivel de participación en los derechos de voto del principal accionista. En este sentido, ante el profundo cambio que está experimentando la normativa relativa a la transparencia de las empresas cotizadas españolas, los resultados obtenidos adquieren una especial relevancia, al poner de manifiesto la necesidad de considerar, en la citado contexto, los incentivos creados por la estructura de propiedad que afectan a la conducta de quienes controlan el proceso de elaboración y divulgación de información contable.

\section{REFERENCIAS}

Andrés Alonso, P.; Azofra Palenzuela, V,. y Rodríguez Sanz, J. A. [2000]: «Endeudamiento, oportunidades de crecimiento y estructura contractual: un contraste empírico para el caso español», Investigaciones Económicas, Vol. 24, n. ${ }^{\circ}$ 3, pp. 641-679.

Azofra, V.; Castrillo, L., y Delgado, M. [2003]: «Ownership concentration, debt financing y the investment opportunity set as determinants of accounting discretion: empirical evidence from Spain», Spanish Journal of Finance $y$ Accounting, n. ${ }^{\circ}$ 115, Special Issue for the 26th Annual Congress. 
Вевсник, L. A. [1999]: «A rent-protection theory of corporate ownership and control», National Bureau of Economic Research, WP 7203.

Bebchuk, L. A.; Kraakman, R., y Triantis, G. G. [2000]: «Stock Pyramids, cross-ownership, and dual class equity», en Concentrated Corporate Ownership, Ed. Randall K. Mork, Chicago.

BeEKes, W.; Pope, P., y Young, S. [2004]: «The Link between earnings timeliness, earnings conservatism and board composition: evidence from the UK», Corporate Governance 12 (I), pp. 47-51.

Berle, A. A., y Means, G. C. [1932]: The modern corporation and private property, Editorial McMillan, New York.

Cheng, Q., y WARFIELD, T. D. [2005]: «Equity Incentives and Earnings Management», The Accounting Review, Vol. 80-2, pp. 441-476.

Christie, A. A.; Joye, M. P., y WatTs, R. L. [2003]: «Decentralization of the firm: theory and evidence», Journal of Corporate Finance, Vol. 9, n. ${ }^{\circ}$ 1, pp. 3-36.

Claessens, S.; DJankov, S., y Lang, L. [2000]: «The separation of ownership and control in East Asian corporations», Journal of Financial Economics, Vol. 58, pp. 81-112.

Demsetz, H. [1983]: «The structure of ownership and the theory of the firm», Journal of Law and Economics, Vol. 26, pp. 375-390.

Demsetz, H., y LeHn, K. [1985]: «The structure of corporate ownership: causes and consequences», Journal of Political Economy, Vol. 93, pp. 1.155-1.177.

DenIS, D. J., y DeNIS, D. K. [1994]: «Majority owner-managers and organizational efficiency», Journal of Corporate Finance, Vol. 1, pp. 91-118.

Dhaliwal, D. S.; Kyung, L., y FARGHer, N. [1991]: «The association between unexpected earnings and abnormal security returns in the presence of financial leverage», Contemporary Accounting Research, Vol. 8, N. ${ }^{\circ} 1$, pp. 20-41.

DycK, A., y Zingales, L. [2004]: «Private Benefits of Control: An International Comparison», The Journal of Finance, Vol. 59, pp. 537-600.

FACCIO, L., y LANG, L. [2002]: «The ultimate ownership of Western European corporations», Journal of Financial Economics, Vol. 65, pp. 365-395.

FAma. E. F. [1980]: «Agency problems and the theory of the firm», Journal of Political Economy, Vol. 88, pp. 288-307.

FAma, E. F., y JensEn, M. C. [1983]: «Separation of ownership and control», Journal of Law \& Economics, Vol. XXVI, pp. 301-325.

FAN, J. P. H., y WoNG, T. J. [2002]: «Corporate ownership structure and the informativeness of accounting earnings in East Asia», Journal of Accounting and Economics, Vol. 33, pp. 401-425.

Francis, J.; SCHIPPER, K., y VINCENT, L. [2005]: «Earnings and dividend informativeness when cash flow rights are separated from voting rights», Journal of Accounting and Economics, Vol. 39-2, pp. 329-360.

Galve GórRiz, C., y Salas Fumás, V. [1992]: «Estructura de propiedad de la empresa española», Revista de Economía Española, n. ${ }^{\circ} 701$, pp. 79-90.

García Lara, J. M., y Mora, A. [2004] «Balance sheet versus earnings conservatism in Europe», European Accounting Review, Vol. 13, pp. 261-292.

García Lara, J. M.; García Osma, B., y Mora, A. [2005] «The effect of earnings management on the asymmetric timeliness of earnings», Journal of Business Finance and Accounting, Vol. 34 (3-4), pp. 691-726.

García Lara, J. M.; García Osma, B., y Peñalva Acedo, F. [2005]: «Evidencia española de la relación entre gobierno corporativo y conservadurismo contable», en Armonización y gobierno de la diversidad (Recurso electrónico): XIII Congreso AECA, Oviedo, 22 a 24 de septiembre de 2005: comunicaciones presentadas. 
García Osma, B., y Gill de Albornoz, B. [2004]: «Las prácticas de buen gobierno en las empresas españolas cotizadas: Efectos sobre la calidad de los estados financieros», Revista de Contabilidad y Tributación, n. ${ }^{\circ}$ 261, pp. 119-158.

González Rodríguez, F., y Menéndez Requejo, S. [1993]: «Implicaciones de la estructura de propiedad sobre las decisiones financieras de la empresa», Revista Europea de Dirección y Economía de la Empresa, Vol. 2, pp. 89-98.

Grossman, S., y HaRT, O. [1988]: «One Share-One Vote and the Market for Corporate Control», Journal of Financial Economics, Vol. 20, pp. 175-202.

GuL, F .A., y WAH, L. K. [2002]: «Insider entrenchment, board leadership structure and informativeness of earnings», Working Paper, disponible en http://ssrn.com/abstract=304399.

HaRRIS, M., y Raviv, A. [1988]: «Corporate Governance: Voting Rights and Majority Rules», Journal of Financial Economics, Vol. 20, pp. 203-35.

Haw, I.; Hu, B.; Hwang, L., y Wu, W. [2004]: «Ultimate Ownership, Income Management, y Legal y Extra-Legal Institutions», Journal of Accounting Research, n. ${ }^{\circ}$ 42, pp. 423-462.

Holderness, C. G., y Sheenan, D. P. [1988]: «The role of majority shareholders in publicy held corporations. An exploratory analysis», Journal of Financial Economics, Vol. 20, pp. 317-346.

Holderness, C. G.; Kroszner, R. S., y Sheehan, D. P. [1999]: «Were the good old days that good? Changes in managerial stock ownership since the great depression», The Journal of Finance, Vol. LIV, pp. 435-469.

Iмноғғ, Е. А., у Lово, G. J. [1992]: «The effect of ex ante earnings uncertainty on earnings response coefficients», The Accounting Review, Vol. 67, pp. 427-439.

Jensen, M. C., y Meckling, W. H. [1976]: «Theory of the firm: managerial behavior, agency cost and ownership structure», Journal of Financial Economic, Vol. 3, pp. 305-360.

Jensen, M. C., y Meckuing, W. H. [1992]: «Specific and general knowledge, and organizational structure», en Contract Economics, WerRIN, L., y WiJKANDER, H. Ed., Blackwell, Oxford, pp. 251-274.

LAfond, R., y Roychowdhury, S. [2006]: «The Implications of Agency Problems between Managers and Shareholders for the Relation between Managerial Ownership and Accounting Conservatism», Working Paper, disponible en http://ssrn.com/abstract=929693.

La Porta, R.; López de Silanes, S.; Shleifer, A., y Vishny, S. (1998): «Law and finance», Journal of Political Economy, Vol. 106, pp. 1.113-1.155.

La Porta, R.; López de Silanes, F., y Shleifer, A. [1999]: «Corporate ownership around the world», The Journal of Finance, Vol. LIV, pp. 471-517.

La Porta, R.; López de Silanes, F.; Shleifer, A., y Vishny, R. [2000]: «Investor protection and corporate governance», Journal of Financial Economics, Vol. 58, n. ${ }^{\circ}$ 1-2, pp. 3-27.

LEUZ, C.; NANDA, D., y WYsocKI, P. [2003]: «Earnings management y investor protection: an international comparison», Journal of Financial Economics, Vol. 27, pp. 505-527.

López IturRiaga, F. J., y Rodríguez Sanz, J. A. [2001]: «Ownership structure, corporate value and firm investment: A simultaneous equations analysis of Spanish companies», Journal of Management and Governance, Vol. 15, pp. 179-204.

Morck, R.; Shleifer, A., y Vishny, R. W. [1988]: «Management ownership and market valuation: an empirical analysis», Journal of Financial Economics, Vol. 20, pp. 293-315.

Peasnell, K. V.; Pope, P. F., y Young, S. [2000]: «Board monitoring y earnings management: Do outside directors influence abnormal accruals?», Working Paper, Lancaster University

Pedersen, T., y Thomsen, S. [1999]: «European patterns of corporate ownership», Journal of International Business Studies, Vol. 28, pp. 759-778.

ShLEIFER, A., y VishnY, R. W. [1986]: «Large shareholders and corporate control», Journal of Political Economy, Vol. 94, pp. 461-488. 
SHLEIFER, A., y VishNY, R. W. [1997]: «A survey of corporate governance», The Journal of Finance, Vol. LII, n. ${ }^{\circ}$, pp. 737-783.

SHLEIFER, A., y Wolfenzon, D. [2002]: «Investor protection and equity markets», Journal of Financial Economics, Vol. 66, pp. 3-27.

Subramanyam, K. R., y WiLD, J. J. [1996]: «Going-Concern status, earnings persistence, and informativeness of earnings», Contemporary Accounting Research, Vol. 13, pp. 251-273.

TEo, S. H., y Wong, T. J. [1993]: «Perceived auditor quality and the earnings response coefficients», The Accounting Review, Vol. 68, pp. 346-367.

WARFIELD, T. D.; WILD, J. J., y WILD, K. L. [1995]: «Managerial ownership, accounting choices, and informativeness of earnings», Journal of Accounting and Economics, Vol. 20, pp. 61-91.

WhiтE, H. [1980]: «A heteroskedasticity-consistent covariance matrix estimator and a direct test for heteroskedasticity», Econometrica, Vol. 48, pp. 817-838.

XIE, B.; DAVIDSON, W. N., y DADALT, P. [2003]: «Earnings management and corporate governance: The role of board and the audit committee», Journal of Corporate Finance, Vol. 9, pp. 295-316.

Yeo, G. H. H.; TAN, P. M. S.; Ho, K. W., y Chen, S. S. [2002]: «Corporate Ownership Structure and the Informativeness of Earnings», Journal of Business Finance \& Accounting, Vol. 29, pp. 1.023-1.046.

ZingaLES, L. [1994]: «The value of the voting right: Study of the Milan stock exchange experience», $R e$ view of Financial Studies, Vol. 7, pp. 125-148. 